\title{
Coarse-grained description of localized inelastic deformation in amorphous metals
}

\author{
Marios D. Demetriou, ${ }^{1, a)}$ William L. Johnson, ${ }^{1}$ and Konrad Samwer ${ }^{2}$ \\ ${ }^{1}$ Keck Engineering Laboratories, California Institute of Technology, Pasadena, California 91125, USA \\ ${ }^{2}$ I. Physikalisches Institut, Universität Göttingen, Friedrich-Hund-Platz 1, 37077 Göttingen, Germany
}

(Received 21 February 2009; accepted 23 April 2009; published online 13 May 2009)

\begin{abstract}
The sequence of shear transformation events that lead to a shear band transition in amorphous metals is described by a spatially random coarse-grained model calibrated to obey the thermodynamic scaling relations that govern flow in a real glass. The model demonstrates that shear banding is a consequence of local shear transformation events that self-organize along planes of maximum resolved shear stress to form extended bands of highly localized deformation. This description suggests that shear band formation is incipient during the early stages of deformation of a randomly inhomogeneous material. (c) 2009 American Institute of Physics.

[DOI: $10.1063 / 1.3133942$ ]
\end{abstract}

Amorphous materials and glasses in general are widely perceived as randomly inhomogeneous structures consisting of nanoscopic heterogeneities with broadly varying properties. ${ }^{1}$ These elemental "defects," which are commonly referred to as shear transformation zones (STZs), are thought to represent regions of atomic cooperativity and believed to be the "carriers" of plastic flow in the glassy structure. ${ }^{2}$ Atomic cooperativity within STZs and its contribution to glass plasticity have been verified by molecular dynamics. ${ }^{3}$ Under strain rates higher than the rate of configurational relaxation, plasticity is understood to propagate through the glassy structure via percolation of STZ softening events biased along planes of maximum resolved shear stress, thereby producing shear bands. Percolation of local instabilities and formation of shear bands are recognized to be the mechanism of plasticity in other randomly inhomogeneous solids, such as highly stochastic foams. ${ }^{4}$ Flow percolation is understood to arise as a consequence of activated hopping across a random configurational potential energy landscape (PEL), as conceived by Stillinger and co-workers ${ }^{5,6}$ and validated by other authors ${ }^{7,8}$ via atomistic simulations. In this letter, the sequence of STZ transitions that lead to shear banding is described by a spatially random coarse-grained model calibrated to obey the thermodynamic scaling relations that govern flow in a real glass. ${ }^{9-14}$

In the present approach, the glassy structure is coarsegrained to the size of the fundamental unit of plasticity, the STZ. Each STZ is assigned an isoconfigurational shear modulus $G$ from a random distribution function, and upon the introduction of an inelastic strain field, the evolution of local $G$ is calculated using a constitutive law that describes the evolution of $G$ during flow. Specifically, by tracking the excess enthalpy of configurational disorder $\Delta H$ stored in each STZ, the associated change in local $G$ is computed according to a balance between softening and relaxation using the average activation barrier for configurational hopping, $W$, as a control parameter. The $\mathrm{Pd}_{43} \mathrm{Ni}_{10} \mathrm{Cu}_{27} \mathrm{P}_{20}$ glass is modeled here, and the experimentally assessed configurational relations for this glass are utilized. ${ }^{10,11,13}$

\footnotetext{
a) Author to whom correspondence should be addressed. Electronic mail: marios@caltech.edu.
}

Constant strain-rate compression is considered at temperature $T=548 \mathrm{~K}$ (as in the experiments of Ref. 14) with a strain rate of $5 \times 10^{-4} \mathrm{~s}^{-1}$ (five times higher than in Ref. 14). This set of conditions is experimentally determined to give rise to inhomogeneous flow (shear banding). Initially the glass is taken to be relaxed at $548 \mathrm{~K}$ (as in Ref. 14). At that temperature, the equilibrium "isoconfigurational" shear modulus of $\mathrm{Pd}_{43} \mathrm{Ni}_{10} \mathrm{Cu}_{27} \mathrm{P}_{20}$ can be estimated to be $G_{e}$ $=G_{g}\left[n\left(1-T / T_{g}\right)\right]=31.6 \mathrm{GPa}$, where $T_{g}=567 \mathrm{~K}$ is the glass transition temperature, $n=1.128$, and $G_{g}=30.4 \mathrm{GPa}^{10}$ The estimated $G_{e}$ is very close to the measured value of 31.5 GPa. ${ }^{14}$ Also, the equilibrium STZ volume at $548 \mathrm{~K}$ can be estimated to be $\Omega_{e}=\Omega_{g}\left(G_{e} / G_{g}\right)=9.5 \mathrm{~nm}^{3}$, where $\Omega_{g}$ $=9.2 \mathrm{~nm}^{3} .{ }^{13}$ Using the average atomic volume of 0.013 $\mathrm{nm},{ }^{3,15}$ the effective number of atoms participating in a STZ transition at $548 \mathrm{~K}$ can be estimated to be $N \approx 700$. No variance in STZ volume is considered.

A cylindrical domain $25 \mathrm{~nm}$ in diameter and $50 \mathrm{~nm}$ in length is coarse grained into tetrahedral elements having volume $\Omega_{e}$ representing individual STZs. Approximately 2600 tetrahedra (STZs) are generated. Initially, each STZ is assigned a random $G$ value from a Gaussian distribution function having mean $G_{e}$ and variance $G_{e} / \sqrt{N}^{16}$ The initial state of the coarse-grained cylinder with randomly varying $G$ is shown in Fig. 1, while the initial Gaussian $G$ distribution is shown in Fig. 2(a).

The STZ softening induced by inelastic deformation is modeled by assuming that the irreversible configurational hopping events of STZs are associated with (1) a buildup of the local configurational enthalpy $\Delta H,(2)$ a decrease in the

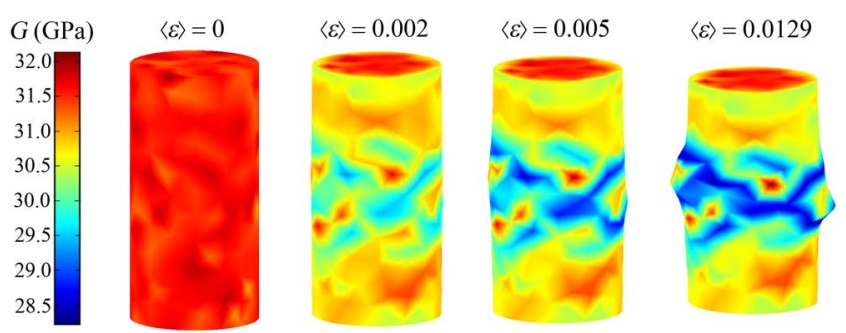

FIG. 1. (Color online) Evolution of the local shear modulus $G$ at different average inelastic strains $\langle\varepsilon\rangle$. The deformation is exaggerated by a factor of 10 for clarity. 


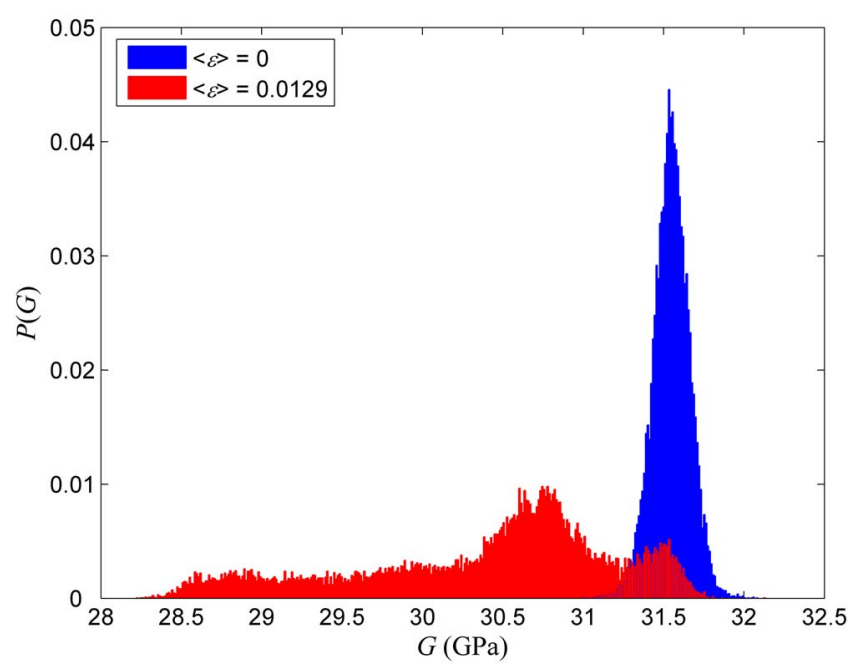

FIG. 2. (Color online) Evolution of the shear modulus distribution from $\langle\varepsilon\rangle=0$ to $\langle\varepsilon\rangle=0.0129$.

local activation barrier for configurational hopping $W$, and (3) a decrease in the local isoconfigurational shear modulus $G$. Assuming that the PEL of a STZ is roughly sinusoidal, ${ }^{9}$ a functional relationship between the average $G$ and $W$ can be derived as $G=G_{g}\left(W / W_{g}\right)^{1 / 2}$, where $W_{g} \equiv\left(8 / \pi^{2}\right) \gamma_{c}^{2} G_{g} \Omega_{g}$ and $\gamma_{c}=0.036$. $^{7,10}$ The rate of softening of a STZ can hence be modeled by tracking the buildup of $\Delta H$ and its effect on $W$ via the following rate equation: ${ }^{10,13}$

$$
\begin{aligned}
\frac{d W}{d t}= & -\left(\frac{2 n W_{g}}{T_{g} \Delta c_{p}}\right)(\eta \dot{\gamma}: \dot{\gamma})-\frac{\left(W-W_{e}\right)\left(W / W_{g}\right)^{1 / 2}}{a \eta / G_{g}} \\
& +\nabla \cdot(D \nabla W) .
\end{aligned}
$$

The first term to the right of Eq. (1) represents the rate of softening (biasing rate) caused by the buildup of configurational enthalpy, and assumes that the inelastic mechanical work is efficiently converted into configurational enthalpy, as has been observed experimentally within the transient inelastic deformation regime. ${ }^{14}$ In this term, $\dot{\gamma}$ is the shear rate tensor, $\eta$ is the viscosity taken to be an inverse Boltzmann distribution function of $W$, i.e., $\eta$ $=\eta_{\infty} \exp \left(W / k_{b} T\right),{ }^{9,12} \eta_{\infty}=5.1 \times 10^{-5} \mathrm{~Pa} \mathrm{~s}$ is the Planck viscosity limit, ${ }^{13}$ and $\Delta c_{p}=2.5 \times 10^{6} \mathrm{MJ} / \mathrm{m}^{3} \mathrm{~K}$ is the heat capacity change at $T_{g}{ }^{17}$ The second term to the right of Eq. (1) represents the relaxation rate (configurational hopping rate) toward an equilibrium barrier $W_{e}$ $=W_{g}\left(G_{e} / G_{g}\right)^{2},{ }^{10,13}$ and assumes a unimolecular Maxwellian relaxation process. Parameter $a$, which for this alloy was determined to be $45.9,{ }^{13}$ quantifies the departure from an ideal relaxation process. In effect, the second term in Eq. (1) describes the rate of local recovery due to relaxation, which in turn implies conversion of configurational enthalpy into thermal energy and heat dissipation. The dissipation process under adiabatic conditions (as in a macroscopic sample) is known to increase temperature giving rise to local thermal softening. For a nanoscopic sample however, like the one considered here, the transport of heat to the isothermal surroundings is expected to occur at a time scale much shorter than the structural relaxation time, resulting in an effectively isothermal process. In the context of this study therefore, the temperature is assumed to remain constant during deformation. The last term in Eq. (1) describes the rate of spatial annihilation, which effectively implies diffusion of configu- rational enthalpy. Such a process is understood to occur by atomic diffusion, with an effective atomic diffusivity approximated by the Stokes-Einstein relation given by $D$ $=k_{B} T / 6 \pi l \eta$, where $l \sim 1.5 \mathrm{~nm}$ is the average atomic size. For small inelastic strains, the last term in Eq. (1) will be significantly smaller than the two preceding terms and thus negligible. However as the inelastic strain increases, this term will become important and will contribute to widen the evolving shear bands (as observed in heavily deformed specimens $^{18}$ ).

Description of transient localized softening during inelastic deformation requires coupling of Eq. (1) with a rate equation that governs the evolution of a conserved strain field. In this study, we utilize one of the simplest sets of strain field equations: the three-dimensional incompressible Navier-Stokes equations. The Navier-Stokes equations govern the evolution of a purely inelastic strain field, essentially ignoring any elastic response preceding the inelastic regime. Nevertheless, since softening is assumed here to be a consequence of inelastic mechanical work being converted into configurational potential energy, a purely inelastic strain field is considered adequate to describe this process. The incompressible form of the Navier-Stokes equations further assumes that the bulk modulus of the material is essentially infinite. The bulk modulus for the state of the material considered here is in fact finite, however, since it will have no bearing on the shear-softening transition simulated here, the incompressible-flow assumption is considered adequate. The set of equations that govern the evolution of the 3D strain field is

$$
\rho\left(\frac{\partial \mathbf{v}}{\partial t}+\mathbf{v} \cdot \boldsymbol{\nabla} \mathbf{v}\right)=(\boldsymbol{\nabla} \cdot \eta \dot{\boldsymbol{\gamma}})-\boldsymbol{\nabla} p \quad(\boldsymbol{\nabla} \cdot \mathbf{v})=0
$$

where $\rho=9340 \mathrm{~kg} / \mathrm{m}^{3}$ is the density, ${ }^{12} p$ is the hydrostatic pressure, and $v$ is the velocity vector. At the loading boundaries, a constant compressive velocity of $12.5 \times 10^{-12} \mathrm{~m} / \mathrm{s}$ is assumed, which corresponds to a constant strain rate of 5 $\times 10^{-4} \mathrm{~s}^{-1}$. Equations (1) and (2) are coupled through $\eta$ $=\eta_{\infty} \exp \left(W / k_{b} T\right)$ and $\dot{\gamma}=\boldsymbol{\nabla} \mathbf{v}+(\nabla \mathbf{v})^{T}$.

The coupled differential equations are integrated numerically using COMSOL. The temporal and spatial evolution of $G$ is presented in Fig. 1. In the initial stages of deformation, the input mechanical work is shown to cause a significant fraction of the STZs to soften. However since local shear transformations are intrinsically biased by the shear component of stress, multiple "soft" paths appear to emerge along planes of maximum resolved shear stress. Percolation ultimately determines a dominant path, along which deformation localizes heavily causing large shear offsets. This process represents the flow pattern that is macroscopically referred to as shear banding. The development of inhomogeneous softening is reflected in the evolution of $G$ distribution, shown in Fig. 2. At the final step of the simulation, the primary peak of the distribution function is shown to shift to lower $G$ values, while a tail reflecting the population of transformed soft STZs develops. Moreover, a peak reflecting the population of inactive "stiff" STZs is maintained in the distribution profile. This finding is consistent with results from recent molecular dynamics simulations, which reveal that the shear transformation is accommodated predominantly by the operation of clusters having shear-prone configurations, while clusters 


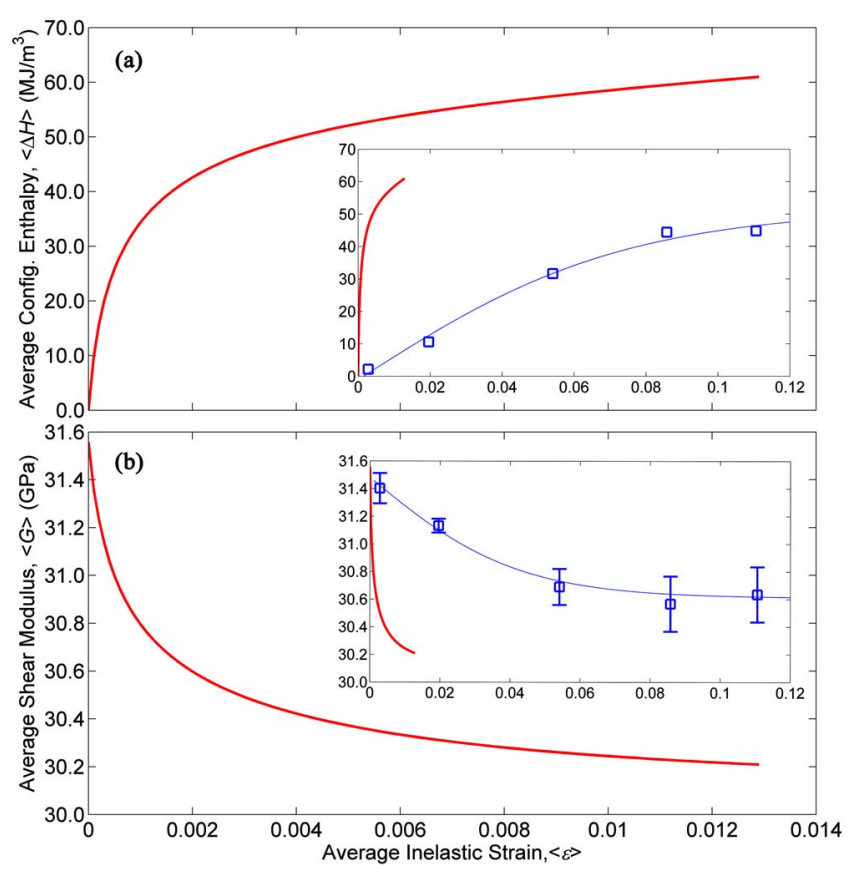

FIG. 3. (Color online) Evolution of (a) the average configurational enthalpy $\langle\Delta H\rangle$ and (b) the average isoconfigurational shear modulus $\langle G\rangle$ vs average inelastic strain $\langle\varepsilon\rangle$. Insets: (top) Evolution of $\langle\Delta H\rangle$ vs $\langle\varepsilon\rangle$ (solid line) and (bottom) $\langle G\rangle$ vs $\langle\varepsilon\rangle$ (solid line) computed in the present study for transient inhomogeneous deformation, plotted together with the experimental data gathered in transient homogenous deformation experiments ( $\square$ ) (Ref. 14). Dashed lines are fits through the experimental data.

having more stable configurations remain mostly inactive during the transition. ${ }^{19,20}$

The instantaneous average configurational enthalpy and isoconfigurational shear modulus during deformation can be computed by integrating over the specimen volume $V$, as follows: $\langle\Delta H\rangle=1 / V \int_{V}\left[\int_{0}^{t}(\eta \dot{\gamma}: \dot{\gamma}) d t\right] d V$ and $\langle G\rangle=1 / V \int_{V} G d V$. $\langle\Delta H\rangle$ and $\langle G\rangle$ are plotted against the average inelastic strain $\langle\varepsilon\rangle$ in Fig. 3. The plots demonstrate that over a global inelastic strain of $\sim 0.01,\langle\Delta H\rangle$ increases toward a nearly steady value of $\sim 60 \mathrm{MJ} / \mathrm{m}^{3}$ while $\langle G\rangle$ decreases from its initial value of $31.6 \mathrm{GPa}$ toward a nearly steady value of $\sim 30.2 \mathrm{GPa}$. In the insets of Figs. 3(a) and 3(b), the simulated evolutions of $\langle\Delta H\rangle$ and $\langle G\rangle$ are plotted together with the evolutions assessed experimentally in homogeneous deformation of $\mathrm{Pd}_{43} \mathrm{Ni}_{10} \mathrm{Cu}_{27} \mathrm{P}_{20}$ at $548 \mathrm{~K}$ and $1 \times 10^{-4} \mathrm{~s}^{-1}$. $^{14}$ As seen in these plots, $\langle\Delta H\rangle$ and $\langle G\rangle$ in inhomogeneous deformation evolve similarly to homogeneous deformation but over considerably smaller inelastic strain $(\sim 0.01$ versus $\sim 0.10$ ), as expected. The model therefore reveals that the structural transitions controlling homogeneous and inhomogeneous deformation are self-similar, but the time scales associated with these transitions are considerably different. By plotting $\langle G\rangle$ against $\langle\Delta H\rangle$ in Fig. 3, a near-linear relation is revealed, which closely matches the trend established by experimental data gathered in $\operatorname{transient}^{14}$ as well as steady-state ${ }^{12}$ homogeneous deformation experiments. This functional relation between shear modulus and stored enthalpy has been determined to be universal for all metallic glasses, ${ }^{13}$ and is believed to represent a scaling law associated with the random PEL features that govern the relaxation and deformation processes of these materials. The agreement of the model with the experimentally established correlation

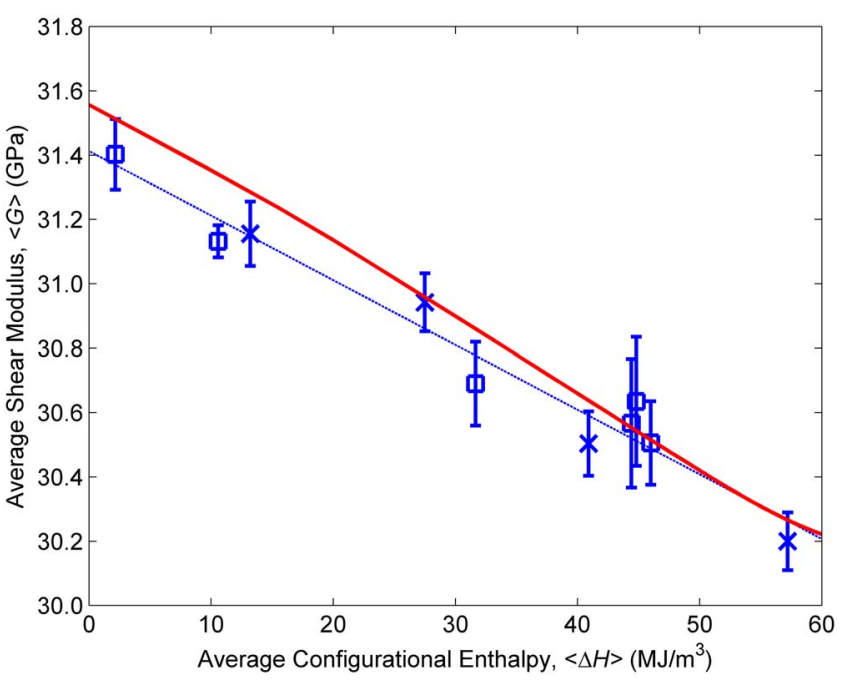

FIG. 4. (Color online) Evolution of the average isoconfigurational shear modulus $\langle G\rangle$ vs average configurational enthalpy $\langle\Delta H\rangle$ computed in the present study for transient inhomogeneous deformation (solid line), plotted together with the experimental data of shear modulus vs stored enthalpy gathered in transient (Ref. 14) $(\square)$ and steady-state (Ref. 12) $(\times)$ homogenous deformation experiments. The dashed line is a fit through the experimental data.

between $\langle G\rangle$ and $\langle\Delta H\rangle$ exemplified in Fig. 4 implies that the input constitutive law, which assumes flow to be a process controlled by the competition between softening and relaxation, is a self-consistent model that effectively obeys the fundamental scaling laws that govern real glasses under flow.

This work was supported in part by the MRSEC Program of the National Science Foundation under Grant No. DMR-0520565.

${ }^{1}$ K. Yoshimoto, T. S. Jain, K. Van Workum, P. F. Nealey, and J. J. de Pablo, Phys. Rev. Lett. 93, 175501 (2004).

${ }^{2}$ A. S. Argon, Acta Metall. 27, 47 (1979).

${ }^{3}$ M. L. Falk and J. S. Langer, Phys. Rev. E 57, 7192 (1998); M. Zink, K. Samwer, W. L. Johnson, and S. G. Mayr, Phys. Rev. B 74, 012201 (2006); C. E. Maloney and A. Lemaitre, Phys. Rev. E 74, 016118 (2006).

${ }^{4}$ M. D. Demetriou, J. C. Hanan, C. Veazey, M. Di Michiel, N. Lenoir, E. Üstundag, and W. L. Johnson, Adv. Mater. (Weinheim, Ger.) 19, 1957 (2007)

${ }^{5}$ F. H. Stillinger and T. A. Weber, Science 267, 1935 (1995).

${ }^{6}$ P. G. Debenedetti and F. H. Stillinger, Nature (London) 410, 259 (2001).

${ }^{7}$ D. J. Lacks and M. J. Osborne, Phys. Rev. Lett. 93, 255501 (2004).

${ }^{8}$ B. A. Isner and D. J. Lacks, Phys. Rev. Lett. 96, 025506 (2006).

${ }^{9}$ W. L. Johnson and K. Samwer, Phys. Rev. Lett. 95, 195501 (2005).

${ }^{10}$ M. D. Demetriou, J. S. Harmon, M. Tao, G. Duan, K. Samwer, and W. L. Johnson, Phys. Rev. Lett. 97, 065502 (2006).

${ }^{11}$ J. S. Harmon, M. D. Demetriou, M. Tao, and W. L. Johnson, Appl. Phys. Lett. 90, 131912 (2007).

${ }^{12}$ J. S. Harmon, M. D. Demetriou, and W. L. Johnson, Appl. Phys. Lett. 90, 171923 (2007).

${ }^{13}$ W. L. Johnson, M. D. Demetriou, J. S. Harmon, M. L. Lind, and K. Samwer, MRS Bull. 32, 644 (2007).

${ }^{14}$ J. S. Harmon, M. D. Demetriou, W. L. Johnson, and K. Samwer, Phys. Rev. Lett. 99, 135502 (2007).

${ }^{15}$ I.-R. Lu, G. P. Görler, and R. Willnecker, Appl. Phys. Lett. 80, 4534 (2002).

${ }^{16}$ L. D. Landau and E. M. Lifshitz, Statistical Physics, Part 1, 3rd ed. (Pergamon, Oxford, 1980), Chap. 12.

${ }^{17}$ X. Hu, Y. Li, S. C. Ng, and Y. P. Feng, Phys. Rev. B 62, 3169 (2000).

${ }^{18}$ Y. H. Liu, G. Wang, R. J. Wang, D. Q. Zhao, M. X. Pan, and W. H. Wang, Science 315, 1385 (2007).

${ }^{19}$ C. E. Lekka, A. Ibenskas, A. R. Yavari, and G. A. Evangelakis, Appl. Phys. Lett. 91, 214103 (2007).

${ }^{20}$ Y. Q. Cheng, A. J. Cao, H. W. Sheng, and E. Ma, Acta Mater. 56, 5263 (2008). 